the Child Psychiatry Branch, NIMH, Bethesda, MD. Tic severity was significantly greater during treatment with relatively high doses of both MPH (20-25 mg bid) and DEX (12.5-22.5 mg bid). Both stimulants significantly decreased hyperactivity and treatment was continued for 1 to 3 years in 14 of 20 patients. Tic exacerbations were reversible, and MPH was better tolerated than DEX. (Castellanos FX, Giedd JN, Elia J, Rapoport JL et al. Controlled stimulant treatment of ADHD and comorbid Tourette's syndrome: effects of stimulant and dose. I Am Acad Child Adolesc Psychiatry May 1997;36:589-596). (Reprints: Dr Castellanos, Bldg 10/Room 6N240, 10 Center Drive, Bethesda, MD 20892).

COMMENT. In treatment of ADHD and TS, the lowest effective dose of stimulant should be used, and increases in dosage should be made slowly. MPH appears less likely to induce persistent tic exacerbation than DEX.

Conventional doses of methylphenidate $(0.1-0.3 \mathrm{mg} / \mathrm{kg})$ produced dramatic improvement in behavior in children with ADHD and tic disorders, but complete normalization of behavior was not attained even with larger doses of $0.5 \mathrm{mg} / \mathrm{kg}$, in a study at the Department of Psychiatry, SUNY at Stony Brook, NY. (Nolan EE, Gadow KD. Children with ADHD and tic disorder and their classmates: behavioral normalization with methylphenidate. I Am Acad Child Adolesc Psychiatry May 1997;36:597-604).

Weinberg WA et al, University of Texas Southwestern Medical Center, Dallas, TX, refer to the depressive reaction induced by methylphenidate, particularly with larger doses and in children with a genetic vulnerability. A stimulant-induced exacerbation of the neurochemical abnormality underlying depression is proposed as an explanation for seizures complicating MPH treatment of ADHD. (I Pediatr April 1997;130:665-669).

\title{
NEUROPATHIES
}

\section{DIABETIC NEUROPATHY IN CHILDREN}

Nerve conduction studies and renal function were evaluated prospectively in 144 diabetic children followed from the time of diagnosis and at 2, 5 and 10 years in the Department of Clinical Neurophysiology, Huddinge University Hospital, Sweden. At diagnosis and before complete remission of the diabetes, abnormal values were found in $25 \%$ of patients for motor conduction velocity and sensory nerve action potentials (SNAP) in the median nerve, for sensory conduction velocity and SNAP in the sural nerve, for parasympathetic autonomic function assessed by R-R intervals in the ECG, and for renal function evaluated by glomerular filtration rate. During long-term follow-up, an initial improved sensory nerve conduction was followed after 2 years by deteriorations in sensory and motor nerve conduction and autonomic nerve function. Poor glycemic control correlated with abnormal prolonged nerve conduction. Improved glycemic control by intense insulin treatment reduced the risk of diabetic neuropathy. (Solders G, Thalme B, Aguirre-Aquino M, et al. Nerve conduction and autonomic nerve function in diabetic children. A 10year follow-up study. Acta Paediatr April 1997;86:361-366). (Respond: Dr G Solders, Dept of Clinical Neurophysiology, Huddinge University Hospital, S-141 86 Huddinge, Sweden).

COMMENT. Optimal glycemic control of diabetes in children may prevent or delay abnormalities in nerve conduction and autonomic dysfunction and the development of diabetic neuropathy. 